\title{
Article \\ Unveiling the Reaction Mechanism of the Das/Chechik/Marek Synthesis of Stereodefined Quaternary Carbon Centers
}

\author{
Pedro J. Silva ${ }^{1,2, *(1)}$ and Carlos E. P. Bernardo ${ }^{1}$ \\ 1 FP-ENAS/Faculdade de Ciências da Saúde, Universidade Fernando Pessoa, Rua Carlos da Maia, 296, \\ 4200-150 Porto, Portugal; cepb_2411@hotmail.com \\ 2 UCIBIO@REQUIMTE, BioSIM, Departamento de Biomedicina, Faculdade de Medicina, Universidade do \\ Porto, 4200-319 Porto, Portugal \\ * Correspondence: pedros@ufp.edu.pt
}

Citation: Silva, P.J.; Bernardo, C.E.P. Unveiling the Reaction Mechanism of the Das/Chechik/Marek Synthesis of Stereodefined Quaternary Carbon Centers. Appl. Sci. 2021, 11, 5002. https://doi.org/10.3390/app11115002

Academic Editor: Sérgio F. Sousa

Received: 13 May 2021

Accepted: 27 May 2021

Published: 28 May 2021

Publisher's Note: MDPI stays neutral with regard to jurisdictional claims in published maps and institutional affiliations.

Copyright: () 2021 by the authors. Licensee MDPI, Basel, Switzerland. This article is an open access article distributed under the terms and conditions of the Creative Commons Attribution (CC BY) license (https:// creativecommons.org/licenses/by/ $4.0 /)$.

\begin{abstract}
The reaction mechanism of the $\mathrm{Cu}^{+}$-catalyzed introduction of two all-carbon-substituted stereocenters in an ynamide system using a Grignard reagent, a zinc carbenoid, and an aldehyde, was investigated using density-functional theory. In contrast to the formation of an organocopper(I) compound and subsequent carbocupration reaction, previously postulated as the initial step, the reaction proved to instead proceed through an initial complexation of the substrate alkyne bond by the $\mathrm{Cu}^{+}$-catalyst, which primes this bond for reaction with the Grignard reagent. Subsequent addition of the zinc carbenoid then enables the nucleophilic attack on the incoming aldehyde, which is revealed as the rate-limiting step. Our computations have also identified the factors governing the regio-and setereoselectivity of this interesting reaction, and suggest possible paths for its further development.
\end{abstract}

Keywords: carbocupration; reaction mechanism; DFT; zinc carbenoid; stereochemistry determinants

\section{Introduction}

Ten years ago, Chechik, Das, and Marek developed a simple method to generate stereodefined quaternary all-carbon-substituted carbon centers in acyclic systems [1]. The strategy relies on the initial formation of a novel $\mathrm{C}-\mathrm{C}$ bond through the addition of an organometallic compound to an ynamide $\mathbf{1}$, a reaction where the required stereocontrol is postulated to be provided by the complexation of the metal atom by the carbonyl moiety of the ynamide (Figure 1). Since the immediate addition of an aldehyde to provide the last substituent in the quaternary carbon is strongly disfavored, the carbometallated ynamide 2 is instead reacted with the Simmons-Smith-Furukawa zinc carbenoid $\left(\mathrm{ICH}_{2} \mathrm{ZnCH}_{2} \mathrm{I}\right)$, yielding a much more reactive allylzinc compound $\mathbf{3}$ which is susceptible to addition of a carbonyl compound to provide the last substituent at the quaternary center. This step is stereocontrolled, presumably through the formation of a Zimmerman-Traxler transition state (a six-membered ring transition state adopting a chair conformation) involving an interaction between the carbonyl oxygen and the zinc atom.

This reaction has been extensively cited since, and was subsequently modified to yield stereo-defined tri-substituted enolates [2]. In this contribution, we analyze the proposed reaction mechanism using computational methods. Our computations characterized the precise nature of the different transition states, identified the rate-determining step, and showed that, instead of the proposed pathway, the initial carbo-metalation event proceeds through the formation of a $\pi$-complex between $\mathrm{CuI}$ and the alkyne, followed by direct methylation of the alkyne bond by the unmodified Grignard reagent. 


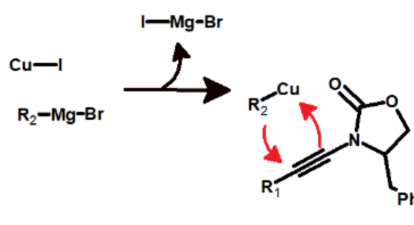

1

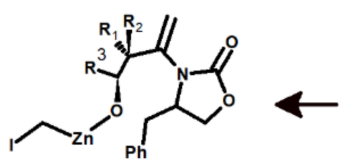<smiles>[2H]C(=C(C)N1C(=O)OCC1Cc1ccccc1)C(C)(C)C</smiles>

2
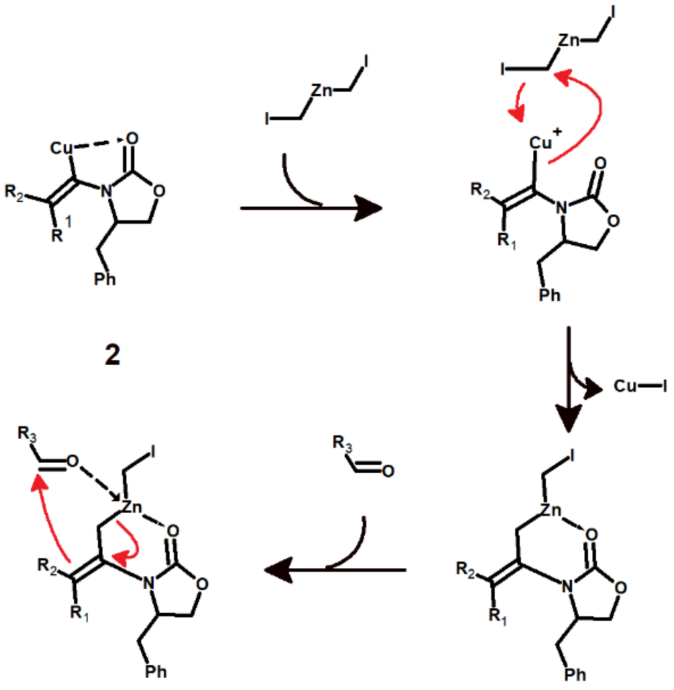

3

Figure 1. Reaction mechanism proposed by Das et al. (adapted from [1]).

\section{Materials and Methods}

The reaction mechanism was investigated using the PBEPW91 functional [3,4], which has been shown to afford good geometric and/or energetic agreement with high-level CCSD(T) or MP2 benchmarking computations in similar $\mathrm{Cu}^{+}$-containing model systems [5,6]. All geometry optimizations were performed using auto-generated delocalized coordinates [7] with the Firefly quantum chemistry package [8], which is partially based on the GAMESS (US) code [9]. The SBKJ pseudo-potential (and associated basis set) [10] was used for $\mathrm{Cu}, \mathrm{Zn}, \mathrm{Br}$, and I. A medium-sized basis set, 6-31G(d), was used for all other elements. Transition states were confirmed to contain a single imaginary frequency. IRC computations confirmed that the obtained transition states did connect the relevant reactant and product states. Zero-point and thermal effects on the free energies at $298.15 \mathrm{~K}$ were computed at the optimized geometries. DFT energies of the optimized geometries obtained with each density functional were then computed using the same functional using 6-311G $(2 \mathrm{~d}, \mathrm{p})$ for all elements except $\mathrm{Cu}$ and $\mathrm{Zn}$ (which used the s6-31G * basis set developed by Swart et al. [11] and the halogens Br and I, which used 6-311G(d,p). Unless otherwise noted, all energy values described in the text include solvation effects in tetrahydrofuran $(\varepsilon=7.6)$ computed using the Polarizable Continuum Model [12-14] implemented in Firefly, as well as dispersion and repulsion interactions with the continuum solvent, which were computed using the method developed by Amovili and Mennucci [15]. Average local ionization energies [16] and distribution of electrostatic potentials on the molecular surface [17] were computed using Multiwfn 3.6 [18]. To facilitate the exploration of a large number of possible pathways, we used a truncated model of the reagent, where $R_{1}$ substituent on the alkyne is a simple methyl group and the bulky phenylmethyl substituent on the oxazolindinone ring was replaced by a methyl group. Numerical simulation of the kinetic behavior predicted by the relative energies of the pre-reactional complexes and transition states was performed using COPASI [19].

\section{Results}

\subsection{Formation of Methylcopper(I)}

In the mechanism proposed be Das et al., the reaction is carried out in tetrahydrofuran (THF) solution. The ynamide first reacts with $\mathrm{CuCH}_{3}$ prepared in situ through the reaction of $\mathrm{CuI}$ with $\mathrm{MgCH}_{3} \mathrm{Br}$. Our DFT computations showed that $\mathrm{CuCH}_{3}$ synthesis from THFcomplexed $\mathrm{CuI}$ and $\mathrm{CH}_{3} \mathrm{MgBr}$ is exergonic by $11.8 \mathrm{kcal} \cdot \mathrm{mol}^{-1}$ and that it proceeds readily with a barrier of only $9.6 \mathrm{kcal} \cdot \mathrm{mol}^{-1}$. It begins through the formation of a pre-reactional 
complex between the THF-solvated $\mathrm{CuI}$ and $\mathrm{CH}_{3} \mathrm{MgBr}$, where the metals and halogens form a quadrilateral arrangement with each halogen about equally bound to each metal and a metal-metal distance of $2.85 \AA$ (Figure 2A). The ensuing electronic arrangement allows the $\mathrm{Mg}-\mathrm{CH}_{3}$ bond to easily vibrate out of the plane formed by the metals and halogens and its distance from $\mathrm{Cu}$ to shorten from $4.64 \AA$ away to $3.02 \AA$. At this point, the transition state is reached and the methyl group effortlessly breaks its bond to $\mathrm{Mg}$ and becomes solely bound to $\mathrm{Cu}$ (Figure 2B). The product of this step contains a $\mathrm{CuCH}_{3}$ loosely bound to the iodine, which then gets completely transferred to the $\mathrm{Mg}$ atom (Figure 2C) at a cost of $1.9 \mathrm{kcal} \cdot \mathrm{mol}^{-1}$.

A)

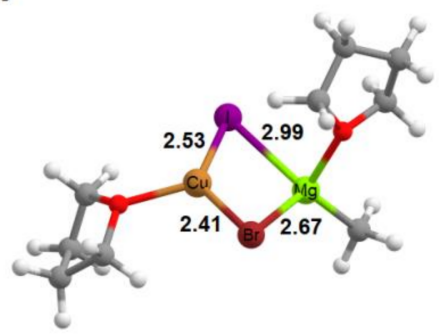

B)

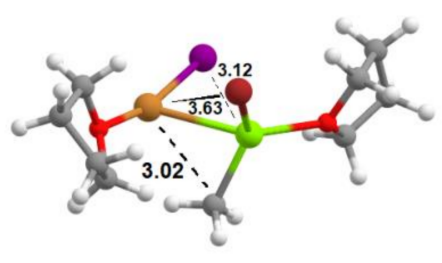

C)

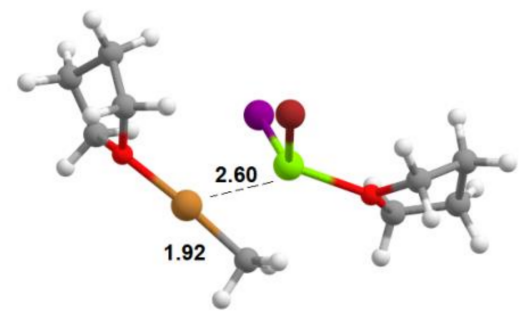

Figure 2. Formation of $\mathrm{CuCH}_{3}$ from $\mathrm{CuI}$ and $\mathrm{MgCH}_{3} \mathrm{Br}$. (A) reactant state; (B) transition state; (C) separated products. Several important bond distances (in $\AA$ ) are highlighted.

\subsection{Carbocupration of the Alkyne Bond}

Addition of the $\mathrm{CuCH}_{3}$ to the ynamide triple bond may subsequently occur in two distinct ways, depending on the orientation of the $\mathrm{Cu}$ atom relative to the ynamide carbonyl (Figure 3). In conformation 1, no interaction between copper and the ynamide oxygen exists, whereas in conformation 2 , the metal atom initially interacts with oxygen through a $2.72 \AA$ bond. Conformation 1 is only slightly more stable (by $2.3 \mathrm{kcal} \cdot \mathrm{mol}^{-1}$ ) than conformation 2. Methyl transfer from $\mathrm{CuCH}_{3}$ to the alkyne may occur from either of these conformations. In both cases, the transition state is reached when the methyl group lies $2.10 \AA$ from the receiving carbon atom. Distances between the methyl group and the $\mathrm{Cu}$ atom hardly differ (by $0.01 \AA$ ) in the transition states arising from each conformation. Activation energies are, in both cases, extremely high (Figure $3 C$ ) and are incompatible with an experimentally viable mechanism, since they translate to reaction rates between $3.5 \times 10^{-12}$ and $2.1 \times 10^{-16} \mathrm{~s}^{-1}$ at $298 \mathrm{~K}$. The addition of the methyl group to the ynamide must therefore follow a different mechanism.

A

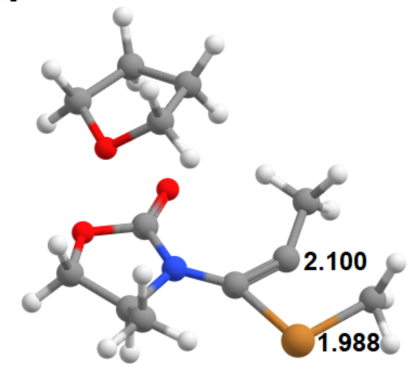

B

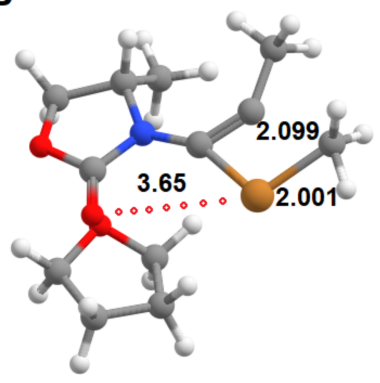

C

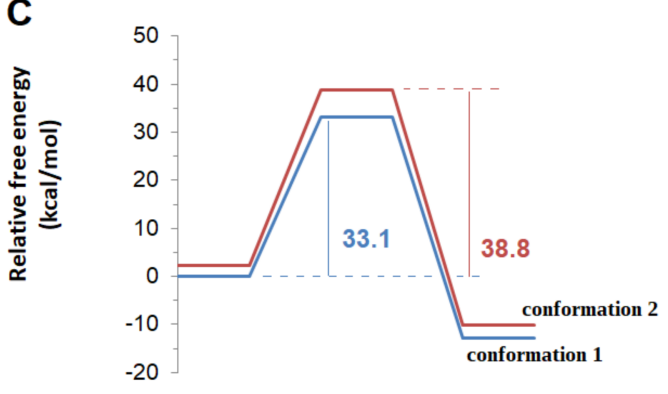

Figure 3. Carbocupration of the ynamide with THF-solvated $\mathrm{CuCH}_{3}$. (A) transition state (conformation 1); (B) transition state (conformation 2); (C) potential energy surface of the carbocupration reaction arising from conformation 1 (blue) and conformation 2 (red). Several important bond distances (in $\AA$ ) are highlighted.

We therefore analyzed the pathways arising from direct complexation of the ynamide substrate with THF-solvated $\mathrm{CuI}$ or $\mathrm{MgCH}_{3} \mathrm{Br}$. Analysis of the solvation energetics 
shows that the free energy change upon solvation of $\mathrm{CuI}$ by a single THF is favorable by $16.3 \mathrm{kcal} \cdot \mathrm{mol}^{-1}$, and that the addition of a second THF molecule to the complex is strongly disfavored because the small decrease in electronic energy afforded by the formation of the new $\mathrm{Cu}-\mathrm{O}$ bond is more than offset by the decrease of system entropy caused by the decrease of molecularity of the system (Figure $4 \mathrm{~A}$ ). $\mathrm{CH}_{3} \mathrm{MgBr}$, in contrast, exists as a complex with two solvent molecules due to the comparable electronic energy decreases entailed by the formation of each of the $\mathrm{Mg}-\mathrm{O}$ bonds, which are in both cases more than enough to offset the entropic losses. The complexation of $\mathrm{MgCH}_{3} \mathrm{Br}$ :2THF with the substrate carbonyl (Figure 4B) proceeds through the ejection of one of the metal-solvating THF molecules and therefore entails an increase in the entropy of the system. The reaction is, however, slightly disfavored, by $4.8 \mathrm{kcal} \cdot \mathrm{mol}^{-1}$, showing that the interaction of $\mathrm{MgCH}_{3} \mathrm{Br}$ with the ynamide carbonyl is weaker than its interaction with the THF molecule that has been released. The $\pi$-complexation of $\mathrm{Cu}^{+}$by the ynamine alkyne bond (Figure $4 \mathrm{C}$ ), in contrast, is exergonic by $10.0 \mathrm{kcal} \cdot \mathrm{mol}^{-1}$ and proceeds without a barrier. It is therefore much more likely for $\mathrm{CuI}$ to complex the ynamide than to undergo the metathesis described above to yield $\mathrm{CuCH}_{3}$. Subsequent addition of $\mathrm{MgCH}_{3} \mathrm{Br}$ to the $\mathrm{Cu}^{+}$-complexed ynamide (Figure 4D) then becomes exergonic (by $0.5 \mathrm{kcal} \cdot \mathrm{mol}^{-1}$ for $\mathrm{MgCH}_{3} \mathrm{Br}: 2 \mathrm{THF}$ and $7.1 \mathrm{kcal} \cdot \mathrm{mol}^{-1}$ for $\mathrm{MgCH}_{3} \mathrm{Br}$ :THF) and yields the reactive ynamide: (CuI:THF):( $\mathrm{MgCH}_{3} \mathrm{Br}$ :THF) complex where direct transfer of the methyl group from the organomagnesium to the alkyne bond can occur. Interestingly, the energetics of the addition of CuI:THF and $\mathrm{MgCH}_{3} \mathrm{Br}$ :THF to the ynamide show that the process is cooperative: ynamide complexation by both metal compounds is exergonic by $17.1 \mathrm{kcal} \cdot \mathrm{mol}^{-1}$, which is higher than the sum of each of the isolated additions of CuI:THF $\left(10.0 \mathrm{kcal} \cdot \mathrm{mol}^{-1}\right)$ and $\mathrm{MgCH}_{3} \mathrm{Br}: \mathrm{THF}\left(1.8 \mathrm{kcal} \cdot \mathrm{mol}^{-1}\right)$ to the bare ynamide. Interaction of each individual metal complex therefore facilitates the addition of the second complex, by triggering an electronic distribution that either facilitates reaction of $\mathrm{CuI}$ with the alkyne bond (in the case of an initial reaction with $\mathrm{MgCH}_{3} \mathrm{Br}$ ) or else renders the carbonyl more nucleophilic to facilitate its reaction with $\mathrm{Mg}^{2+}$ (when CuI adds to the alkyne bond). Analysis of the geometry of the CuI-complexed (or Mg-complexed) ynamides (Figure 4B,C) suggests that this effect is at least partly due to the lower ability of the lone pair of the nitrogen losing the ability to resonate with the alkyne $\pi$-bonds in both situations: in the $\mathrm{Cu}^{+}$-alkyne complex, the $\pi$-bond which (in the free ynamide) partially overlaps with the lone pair orbital now interacts mostly with CuI, and the remaining alkyne $\pi$-bond lies almost perpendicular to the nitrogen lone pair orbital. The lone pair can therefore resonate more strongly with the carbonyl, leaving its oxygen atom more nucleophilic and enabling a better bond with the $\mathrm{Mg}^{2+}$. Conversely, in the $\mathrm{Mg}^{+}$-complexed ynamide this electron pair has partly delocalized into the $\mathrm{N}-\mathrm{C}=\mathrm{O}$ moiety, which decreases the HOMO-LUMO gap and facilitates subsequent attack by $\mathrm{CuI}$.

\subsection{Analysis of the Regioselectivity of Methyl Transfer}

The methyl group may be transferred to either end of the alkyne bond. To maintain consistency with the nomenclature common in the field [20,21], we will refer to the methyl addition to the heteroatom-substituted alkyne carbon as "the branched reaction", and refer to the methyl addition to the other end as "the linear reaction". Analysis of the potential energy surface reveals that the transition state (TS1) leading to the experimentally-obtained "linear" intermediate Int1 (Figure 5A) is $17.6 \mathrm{kcal} \cdot \mathrm{mol}^{-1}$ more stable than the transition state (TS1') leading to the "branched" isomer (Int1') (Figure 5B). The transition states differ most markedly in the length of the bonds between $\mathrm{Cu}^{+}$and the alkyne bond atoms and in the presence or absence of a $\mathrm{Mg}^{2+}$-carbonyl bond (which is not compatible with the short distance between the organometallic methyl and the ring-proximal alkyne carbon atom in TS1'). The reaction products arising from these transition states differ in energy by a non-negligible amount $\left(8.6 \mathrm{kcal} \cdot \mathrm{mol}^{-1}\right)$, in spite of having very similar $\mathrm{Mg}-\mathrm{O}$ and $\mathrm{Cu}^{+}$-alkyne distances. This suggested that other factors govern the relative stabilities of the "linear" and "branched" isomers. Analysis of the energies of the different fragments in these intermediates (the organic moiety, CuI-THF and MgBr-THF) shows that most of the 
extra stability of the linear isomer is due to the higher stability of the linear organic moiety, rather than (as postulated earlier) on the existence of more favorable interactions between the organic portion and the metals in the linear isomer (Table 1). Those interactions are, however, crucial in the kinetic preference of the "linear" pathway over the pathway leading to the "branched" product.

A

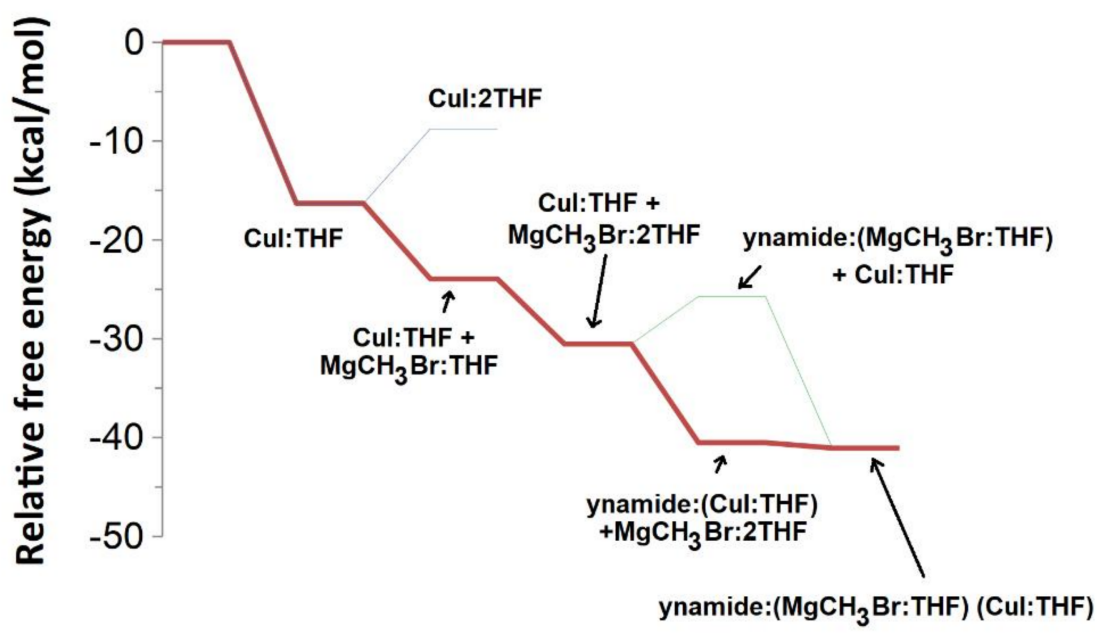

B
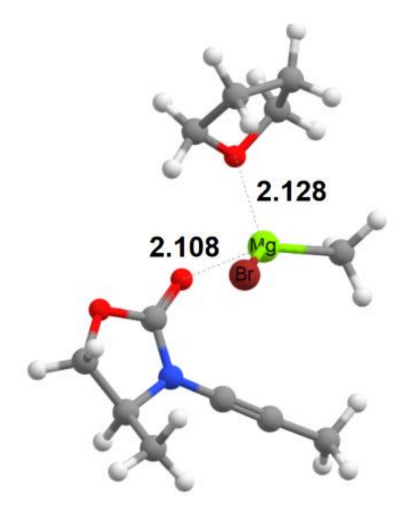

C

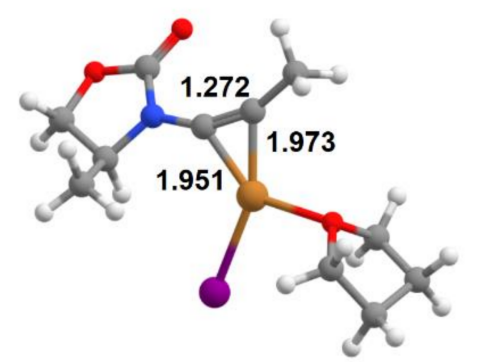

D

Figure 4. Addition of THF-solvated $\mathrm{MgCH}_{3} \mathrm{Br}$ or $\mathrm{CuI}$ to the ynamide. (A) potential energy surface (starting from infinitely separated THF, $\mathrm{CuI}, \mathrm{MgCH}_{3} \mathrm{Br}$, and ynamide) of the solvation of each metallic reagent and subsequent reactions with ynamide; (B) ynamide: ( $\mathrm{MgCH}_{3} \mathrm{Br}$ :THF); (C) ynamide: (CuI:THF); (D) ynamide: $\left(\mathrm{MgCH}_{3} \mathrm{Br}: \mathrm{THF}\right)$ :(CuI:THF). Several important bond distances (in $\AA$ ) are highlighted.

\subsection{Homologation of Intermediate 1 with $\mathrm{ICH}_{2} \mathrm{ZnCH}_{2} \mathrm{I}$}

Intermediate 1 is very crowded around the $\mathrm{C}=\mathrm{C}$ double bond (Figure $6 \mathrm{~A}$ ). Addition of other substituents to this bond to generate the desired quaternary compound must therefore await the ejection of the $\mathrm{MgBr}$ moiety. This step is performed through the intervention of the THF solvent molecules, which will replace the carbonyl as $\mathrm{Mg}^{2+}$ ligands. In a system containing a truncated model of Intermediate 1 (where the CuI-complexed moiety has been removed to decrease computational cost) with four extra THF molecules, $\mathrm{MgBr}$ removal proceeds readily through simple elongation of the $\mathrm{Mg}^{2+}$-carbonyl bond from $2.18 \AA$ to $2.66 \AA$. At this point $\left(11.7 \mathrm{kcal} \cdot \mathrm{mol}^{-1}\right.$ above the initial state) the transition state is reached and the $\mathrm{Mg}^{2+}$-ynamide bond breaks, whereupon a hexagonal $\mathrm{Mg}^{2+} \mathrm{Br}: 5 \mathrm{THF}$ species is 
released. This reaction is readily reversible because the end species is only $0.5 \mathrm{kcal} \cdot \mathrm{mol}^{-1}$ more stable than the initial state.

A)

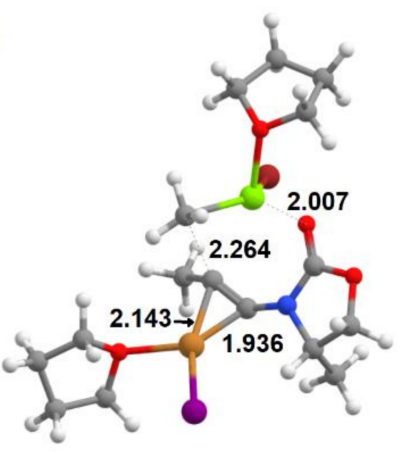

B)

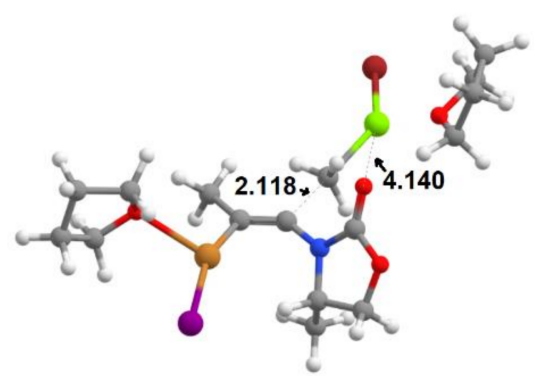

C)

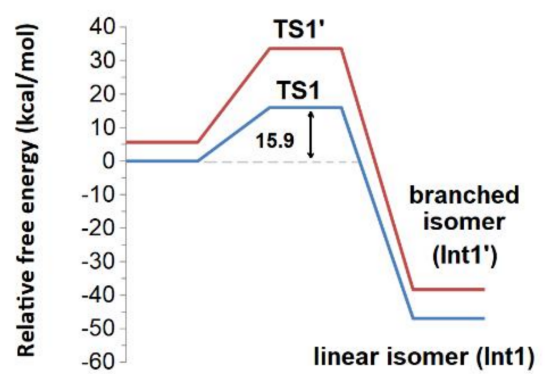

Figure 5. Regioselectivity of addition of THF-solvated $\mathrm{MgCH}_{3} \mathrm{Br}$ to CuI:ynamide. (A) transition state (TS1) of the formation of the linear isomer; (B) transition state (TS1') of the formation of the branched isomer; (C) potential energy surface of the formation of each regioisomer. Several important bond distances (in $\AA$ ) are highlighted.

Table 1. Electronic energy $\left(\mathrm{kcal} \cdot \mathrm{mol}^{-1}\right)$ differences (including solvation effects in THF) between the individual fragments of linear and branched isomers of intermediate 1, computed with the PBEPW91 density-functional. Computations of the energies of the individual fragments have been performed at the same geometry as that in the full molecule. Negative values denote preference for the "linear" over the "branched" isomer.

\begin{tabular}{cccc}
\hline & Reactant State & Transition State & Product \\
\hline Mg moiety & 0.6 & 5.8 & -2.0 \\
\hline CuI moiety & 0.0 & -1.0 & 2.2 \\
\hline Mg-organic moiety & -2.8 & -21.6 & -4.8 \\
\hline CuI-organic moiety & -0.2 & -7.1 & -10.5 \\
\hline organic moiety & -0.1 & 1.7 & -10.1 \\
\hline Full complex & -3.0 & -17.7 & -10.3 \\
\hline
\end{tabular}

The attack of this Mg-free intermediate (Int1) complexed by CuI by the SimmonsSmith-Furukawa zinc carbenoid $\left(\mathrm{ICH}_{2} \mathrm{ZnCH}_{2} \mathrm{I}\right)$ may proceed from different directions, which can be (roughly) defined by the position of the carbenoid relative to the nucleophilic $\mathrm{C}=\mathrm{O}$ moiety on the oxazolidinone ring and by the dihedral angle between the $\mathrm{C}=\mathrm{C}$ bond and the plane of the ring. Since this vast array of possibilities considerably complicates the description of the results, we will first analyze the formation of intermediate 2 through each of the attack vectors, and then discuss the possible routes of interconversion between the different forms of intermediate 2 .

\subsubsection{Attack through the Direction Opposite the Oxazolidinone Carbonyl}

The pre-reactional complex arising from the attack through the face opposite the oxazolidinone carbonyl (Figure 6B) is appreciably more stable (by $10.5 \mathrm{kcal} \cdot \mathrm{mol}^{-1}$ ) than the infinitely-separated reactants due to the electrostatic attraction between the positivelycharged $\mathrm{Zn}^{2+}$ and the anionic intermediate 1. Surprisingly, our exploration of the subsequent potential energy surface showed that the $\mathrm{CH}_{2}$ transfer from the zinc carbenoid to the $\mathrm{C}=\mathrm{C}$ bond in this case takes place through the direct intervention of $\mathrm{Cu}^{+}$(Figure $6 \mathrm{C}$ ). This preference can be rationalized by examination of the average local ionization energies (Figure 7A) of the Int2 intermediate, which feature an absolute minimum (i.e., highest nucleophilic character) on the $\mathrm{Cu}^{+}$, which will therefore be the location with the highest propensity to attack the relatively electrophilic $\mathrm{CH}_{2}$ group (Figure $7 \mathrm{~B}$ ) on $\mathrm{ICH}_{2} \mathrm{ZnCH}_{2} \mathrm{I}$. 
In the transition state, located only $10.7 \mathrm{kcal} \cdot \mathrm{mol}^{-1}$ above the pre-reactional complex, the bond order of the $\mathrm{C}-\mathrm{I}$ bond has decreased to 0.45 , and a half-bond has formed between the $\mathrm{CH}_{2}$ and $\mathrm{Cu}^{+}$group. The collapse of this transition state is then effected through complete transfer of the loosely-bound iodine to the $\mathrm{Zn}^{2+}$ and formation of a new $\mathrm{C}-\mathrm{C}$ bond between $\mathrm{CH}_{2}$ and the hetero-substituted carbon in the $\mathrm{C}=\mathrm{C}$ double bond. This is accompanied by a partial migration of $\mathrm{Cu}^{+}$to the other carbon of the double bond and by reduction of $0.5 \mathrm{in}$ the total bond orders of each fragment. Analysis of Löwdin charge variations shows that the $\mathrm{ZnICH}_{2} \mathrm{I}$ moiety present in the product now contains 0.69 electrons more than in the beginning, of which 0.46 electrons originate in the Int 2 moiety and 0.23 have come from the transferred $\mathrm{CH}_{2}$.

A)

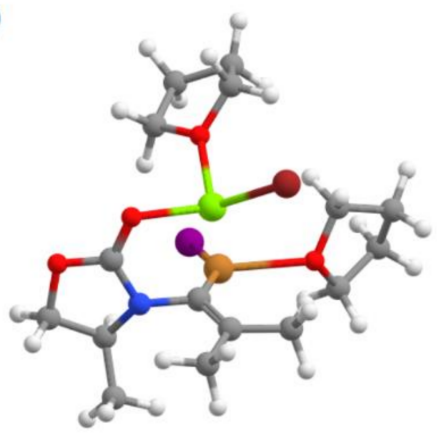

D)

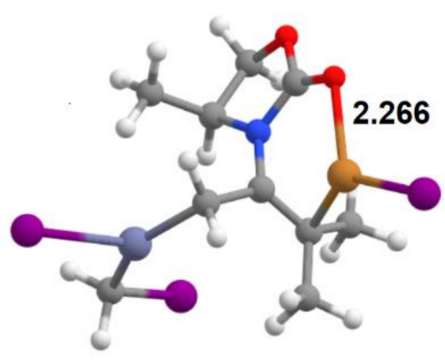

B)

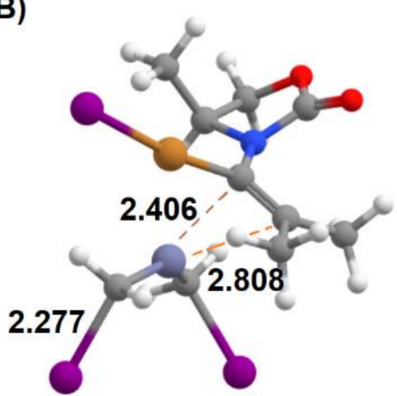

C)

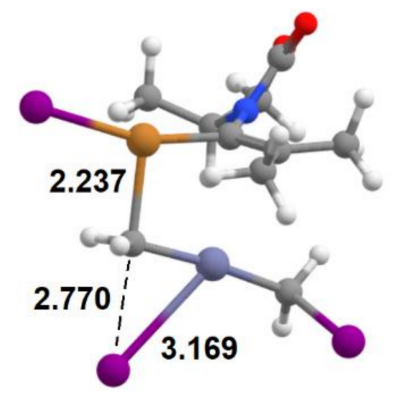

E)

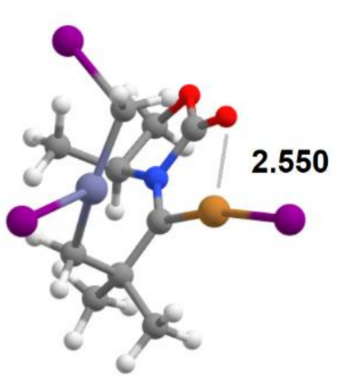

F)

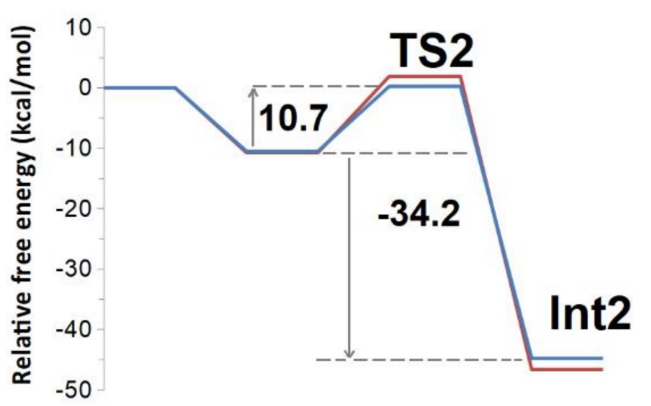

Figure 6. Important structures in the pathway towards intermediate 2 (Int2). (A) Int1:(CuI:THF):(MgBr:THF); (B) Int1:CuI $+\mathrm{ICH}_{2} \mathrm{ZnCH}_{2} \mathrm{I}$ pre-reactional complex; (C) TS2; (D) Int2; (E) Int2'; (F) Potential energy surface of the zinc homologation of Mg-free Int1. The blue line refers to the attack from the "un-encumbered" direction (leading to isomer " $\alpha$ " of Int2), whereas the red line describes homologation from the "encumbered" reaction, leading to isomer " $\beta$ " of Int2.

Examination of the evolution of the bond orders along the reaction coordinate leading from the transition state to Int2 (Figure 8) shows that this rearrangement occurs in three stages: first the bond order between $\mathrm{CH}_{2}$ and I decreases, concurrently with a simultaneous increase of the bond orders of the $\mathrm{Cu}-\mathrm{CH}_{2}$ and $\mathrm{Zn}-\mathrm{I}$ bonds. Then, as the $\mathrm{CH}_{2}-\mathrm{I}$ bond completely disappears, the bond orders of both the $\mathrm{Cu}-\mathrm{CH}_{2}$ bond and the bond between $\mathrm{Cu}$ and the heterosubstituted ynamide carbon decrease in similar amounts to enable the formation of the bond between $\mathrm{CH}_{2}$ and the heterosubstituted carbon. This stage is accompanied by a decrease in the double-bond character of the formal $\mathrm{C}=\mathrm{C}$ bond in ynamide. Finally, the remnants of the $\mathrm{Cu}-\mathrm{CH}_{2}$ bond are replaced by the formation of a half-bond between $\mathrm{Cu}$ and the non-heterosubstituted carbon of the $\mathrm{C}=\mathrm{C}$ bond, simultaneously with the further loss of some of its double-bond character. This complex electronic rearrangement occurs spontaneously without additional transition states, and yields a very stable intermediate (Int2) that lies $34.2 \mathrm{kcal} \cdot \mathrm{mol}^{-1}$ below the pre-reactional complex. The alternative formation of an intermediate bearing the new $\mathrm{CH}_{2}$ group on the opposite carbon (Int2') is thermodynamically much less favored, since Int $2^{\prime}$ lies $22.3 \mathrm{kcal} \cdot \mathrm{mol}^{-1}$ above Int2. Separate analysis of the energies of the individual fragments (Table 2) shows that the relative instability of Int2' is not due to geometric constrains that affect the stabilization of the $\mathrm{Zn}$-containing 
moiety by the oxazolidinone, but to the intrinsically higher energy of the organic moiety of Int2' (compared to that of Int2).

\section{Int2}

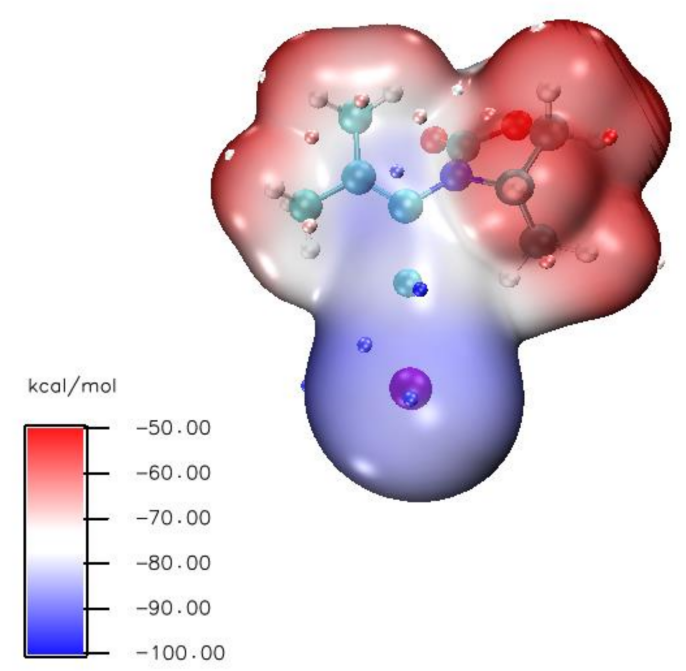

\section{$\mathrm{Zn}\left(\mathrm{CH}_{2}\right)_{2}$}

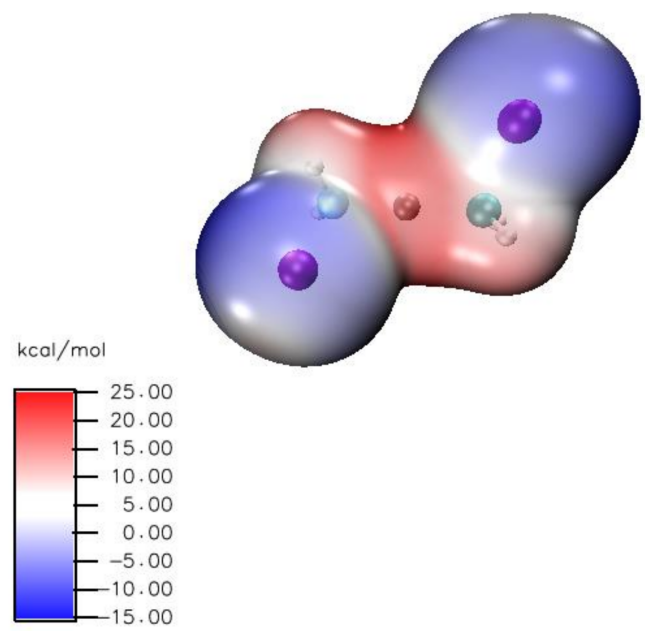

Figure 7. Electrostatic potentials mapped on the density isosurfaces of Int2 (0.001 a.u.) and $\mathrm{ICH}_{2} \mathrm{ZnCH}_{2} \mathrm{I}(0.001$ a.u.). For Int2, the minima (maxima) of the average localized ionization energy are shown as blue (red) dots superposed on the density isosurface.

Table 2. Electronic energies including solvation effects in THF (in $\mathrm{kcal} \cdot \mathrm{mol}^{-1}$ ) of individual fragments of intermediates 2 and $2^{\prime}$, computed with the PBEPW91 density-functional. Computations of the energies of the individual fragments have been performed at the same geometry as that in the full molecule. The electron pair in the severed bond between the organic moiety and $\mathrm{ZnI}\left(\mathrm{CH}_{2} \mathrm{I}\right)$ was divided symmetrically between the fragments.

\begin{tabular}{cccc}
\hline & Int2 & Int2 $^{\prime}$ & Int2-Int2 $^{\prime}$ \\
\hline organic moiety & $-348,955.2$ & $-348,892.7$ & -62.5 \\
organic moiety + CuI & $-478,913.8$ & $-478,885.5$ & -28.3 \\
$\mathrm{ZnI}\left(\mathrm{CH}_{2} \mathrm{I}\right)$ & $-180,031.3$ & $-180,030.3$ & -1.1 \\
Full molecule & $-659,304.2$ & $-659,283.0$ & -21.3 \\
interaction between $\mathrm{ZnI}\left(\mathrm{CH}_{2} \mathrm{I}\right)$ and organic-CuI & -359.2 & -367.2 & 8.1 \\
\hline
\end{tabular}

Two products of the attack on Int 1 are possible (Int2 $\alpha$ and Int $2 \beta$ ) depending on the direction of approach of the Simmons-Smith-Furukawa zinc carbenoid relative to the plane formed by the $\mathrm{C}=\mathrm{C}$ bond and the coordinating CuI. Those attacks are not equally favored because in one of them (which we will designate as "attack from below the plane of the oxazolidine ring", or "encumbered") the attacking zinc carbenoid may clash with the substituent on the oxazolidinone ring (methyl, in our models, or the bulkier phenylmethyl in the experimental setup). Our computations reveal that, indeed, when the attack proceeds from below the ring plane, the steric interaction of the ring substituent with the zinc carbenoid increases the transition state energy by a modest amount $\left(1.6 \mathrm{kcal} \cdot \mathrm{mol}^{-1}\right)$. Although the reaction is thermodynamically more favorable (by $1.8 \mathrm{kcal} \cdot \mathrm{mol}^{-1}$ ) than in the attack from the direction above the ring, the high spontaneity of the reaction (exergonic by more than $34 \mathrm{kcal} \cdot \mathrm{mol}^{-1}$ in both cases) entails that the reverse reactions will have too large an activation energy and the reaction outcome will therefore be kinetically-controlled (rather than thermodynamically-controlled), leading to a non-racemic mixture of the two products where the one arising from the attack of Int1 by the zinc compound from the "un-encumbered" direction (Int2 $\alpha$ ) will be predominant (Figure 6F). 
A)

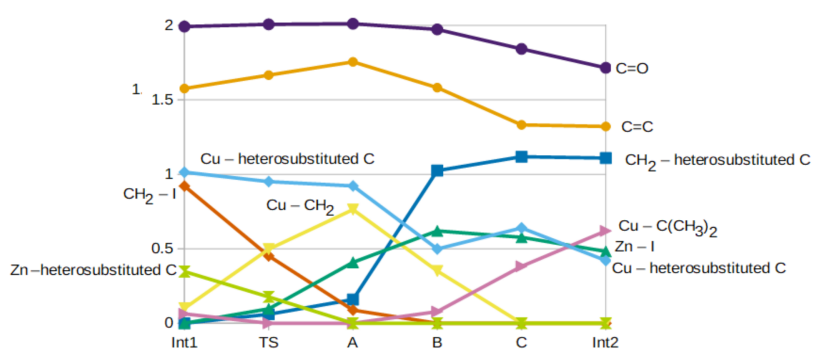

\section{B)}

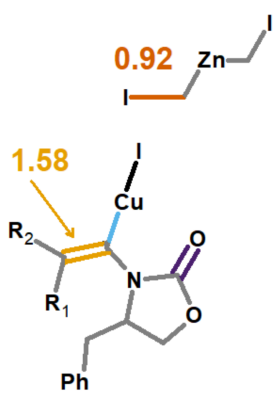

Int1 + $\mathrm{Zn}\left(\mathrm{CH}_{2} \mathrm{l}\right)_{2}$

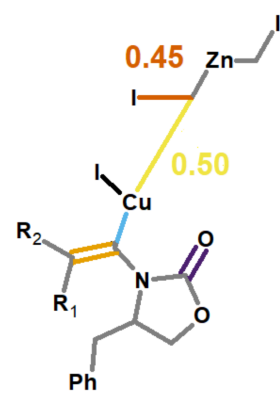

TS

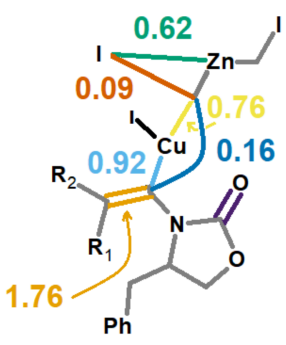

A

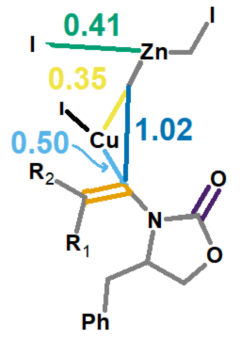

B

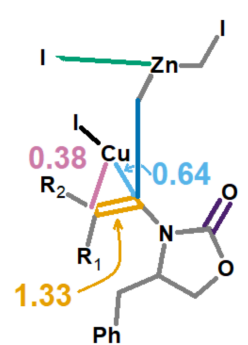

C

Figure 8. Electronic rearrangements from Int1 to Int2. (A) Evolution of bond orders of key chemical bonds along the reaction coordinate; (B) Schematic structures along the reaction coordinate. Each bond is colored according to the palette in the graph in panel A. Structures A, B, and C are located (respectively) 2.5, 31.7, and $43.2 \mathrm{kcal} \cdot \mathrm{mol}^{-1}$ below the transition state, on the reaction coordinate leading to Int2. Selected bond orders are shown.

\subsubsection{Attack Proceeding through Zn Coordination by the Oxazolidinone Carbonyl}

The pre-reactional complex arising from complexation of the zinc carbenoid species by the oxazolidinone ring (Figure 9A) is slightly less favored than the pre-reactional complex described in the previous section. It affords, however a more favorable path to the transfer of $\mathrm{CH}_{2}$ to Int1, with barriers as low as $0.6 \mathrm{kcal} \cdot \mathrm{mol}^{-1}$ (when approaching from the direction above the plane of the ring) or $7.2 \mathrm{kcal} \cdot \mathrm{mol}^{-1}$ (when approaching from the opposite direction). As in the path described in the previous section, $\mathrm{CH}_{2}$ is attacked by the excess electron density in $\mathrm{Cu}^{+}$, with concomitant weakening of the $\mathrm{CH}_{2}-\mathrm{I}$ bond and migration of this iodide to $\mathrm{Zn}$ (Figure 9B). However, in contrast to that path, subsequent $\mathrm{CH}_{2}$ transfer to the $\mathrm{N}$-heterosubstituted carbon in the $\mathrm{C}=\mathrm{C}$ bond is now accompanied by the detachment of $\mathrm{Cu}$ from this bond, and its reattachment to the transferred $\mathrm{CH}_{2}$ (Figure 9C). A facile $\mathrm{Cu}$-migration from $\mathrm{CH}_{2}$ to the non-heterosubstituted carbon in the $\mathrm{C}=\mathrm{C}$ bond (Figure 10) quickly converts this intermediate ( $\left.\mathrm{Int} 2 \mathrm{CuCH}_{2}\right)$ into Int2 isomers where (as in the Int $2 \alpha$ and Int2 $\beta$ described above) $\mathrm{Zn}$ remains bound to the transferred $\mathrm{CH}_{2}$ group and $\mathrm{Cu}$ is bound to the $C=C$ bonded atoms, and which can be distinguished from Int $2 \alpha$ and Int $2 \beta$ by the existence of stabilizing interactions between $\mathrm{Zn}$ and the oxazolidinone ring. Numerical simulation of the kinetic behavior predicted by the relative energies of the pre-reactional complexes and transition states of their conversion into Int 2 intermediates confirmed that, despite their slightly higher energy, the pre-reactional complexes where $\mathrm{Zn}^{2+}$ interacts with the carbonyl oxygen intermediates have a dominant kinetic role, because of the very small barriers of their associated transition states. Accordingly, the resulting products (Int2 $\delta$ and Int $2 \gamma$ ) will each be two to three times more abundant than Int $2 \alpha$, and Int $2 \beta$ (which arises from the most stable pre-reactional complex) will be residual due to its transition 
state having a larger energy than the one required for complete reagent separation (and subsequent recombination into the pre-reactive complexes leading to the other isomers).

A)

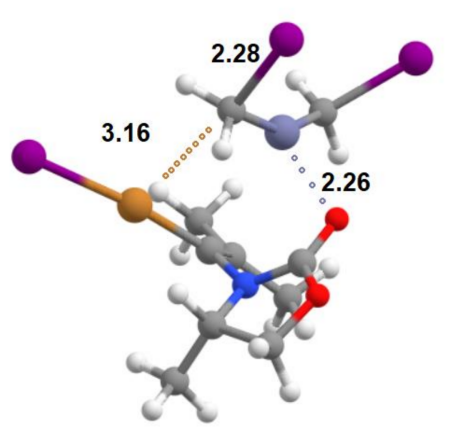

C)

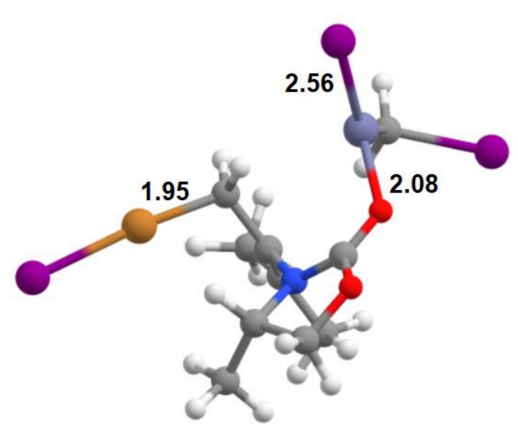

B)

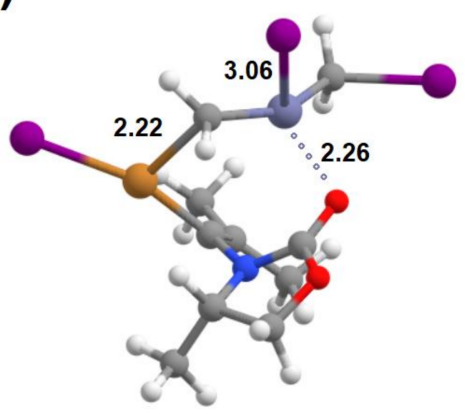

D)

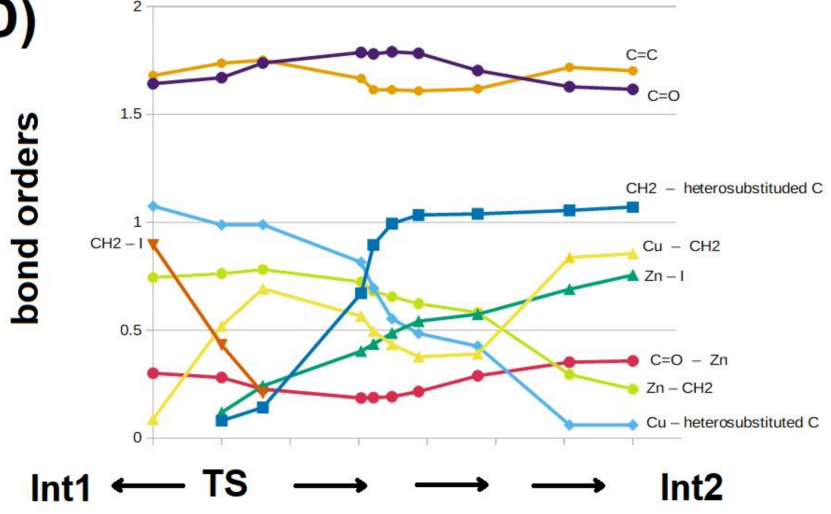

Figure 9. Important structures in the pathway towards intermediate 2 ( $\mathrm{Int} 2 \mathrm{CuCH} 2)$ arising from attack through the carbonyl side of the ring. (A) pre-reactional complex; (B) TS2; (C) Int2 $\mathrm{CuCH}_{2}$; (D) changes in bond order on the pathway from pre-reactional complex to $\mathrm{Int} 2 \mathrm{CuCH} 2$.

A)

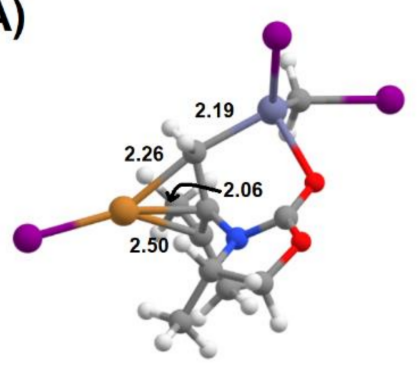

B)

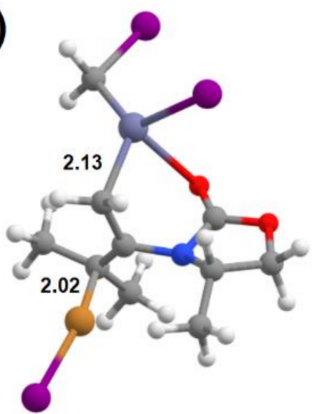

C)

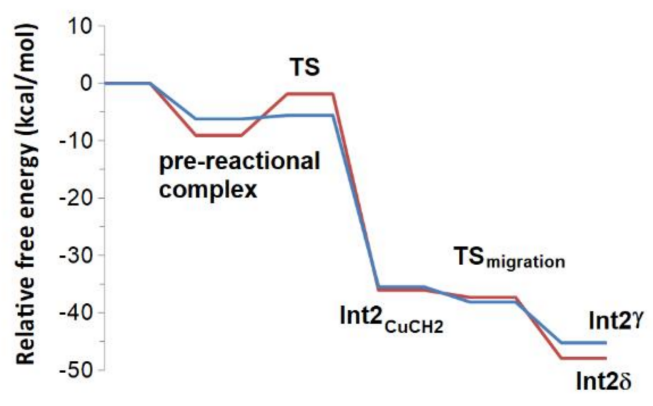

Figure 10. Further structures on the pathway from Int2 $\mathrm{CuCH} 2$ to Int2. (A) Cu migration transition state; (B) Int2 $\gamma$; (C) potential energy surface of the pathway from Int1 to Int2 $\mathrm{CuCH} 2$ to Int2. The blue line refers to the attack from above the ring plane (leading to isomer $\gamma$ of Int2), and the red line to an attack below the plane of the ring (leading to isomer $\delta$ of Int2).

\subsection{Simultaneous Generation of Two Stereocenters through Addition of Aldehyde to Int2}

Each of the four possible Int2 species may now perform a nucleophilic attack on an incoming aldehyde. This attack is aided by the $\mathrm{Zn}^{2+}$ moiety, which binds to the carbonyl in the incoming aldehyde, thereby increasing the electrophilicity of the carbonyl carbon atom and stabilizing the negative charge that accumulates on the carbonyl oxygen atom: in the 
absence of this coordination, the attack has an activation free energy of $32.2 \mathrm{kcal} \cdot \mathrm{mol}^{-1}$, rather than the $18.8-27.9 \mathrm{kcal} \cdot \mathrm{mol}^{-1}$ observed otherwise (Table 3).

Table 3. Activation free energies in THF (in $\mathrm{kcal} \cdot \mathrm{mol}^{-1}$ ) and key geometric parameters of the transition states linking the different Int 2 isoforms to products with two quaternary stereocenters. The configuration at the stereocenter arising from the alkyne is shown first, followed by that of the stereocenter arising from the acetaldehyde. For the configuration assignment of the alkyne carbon, (where, for computational expediency, we had two identical methyl groups) we assigned the lowest priority to the methyl group originally present as the alkyne substituent and the second-lowest to the methyl group donated by the organometallic reagent.

\begin{tabular}{|c|c|c|c|c|}
\hline $\begin{array}{l}\text { Int2 Isoform } \\
\text { Reacting with } \\
\text { Acetaldehyde }\end{array}$ & $\begin{array}{c}\text { Product } \\
\text { Configuration }\end{array}$ & $\begin{array}{c}\text { Activation Free } \\
\text { Energy } \\
\left(\mathrm{kcal} \cdot \mathrm{mol}^{-1}\right)\end{array}$ & $\begin{array}{c}\mathrm{C}=\mathrm{O} \cdots \mathrm{Zn} \\
\text { Distance }(\AA)\end{array}$ & $\begin{array}{c}\mathrm{C} \cdots \mathrm{C}=\mathrm{O} \\
\text { Distance }(\AA)\end{array}$ \\
\hline Int $2 \alpha$ & $(S)(S)$ & 20.2 & 2.07 & 2.29 \\
\hline Int $2 \alpha$ & $(S)(R)$ & 20.7 & 2.04 & 2.30 \\
\hline Int $2 \gamma$ & $(S)(S)$ & 19.8 & 2.02 & 2.06 \\
\hline Int $2 \gamma$ & $(S)(R)$ & 18.8 & 2.02 & 2.02 \\
\hline Int $2 \beta$ & $(R)(R)$ & 26.9 & 2.04 & 2.25 \\
\hline Int $2 \beta$ & $(R)(S)$ & 27.9 & 2.04 & 2.28 \\
\hline Int $2 \delta$ & $(R)(R)$ & 24.9 & 2.05 & 2.32 \\
\hline Int $2 \delta$ & $(R)(S)$ & 23.1 & 2.04 & 2.33 \\
\hline
\end{tabular}

Interestingly, the position of the $\mathrm{Cu}^{+}$ligand in the transition states arising from Int $2 \delta$ and Int $2 \gamma$ is very similar to that in their Int $2 \mathrm{CuCH} 2$ precursors (as shown in Figure 11, panels $\mathrm{C}$ and $\mathrm{E}$ ), because complexation of the acetaldehyde carbonyl by the $\mathrm{Zn}^{2+}$ releases the $\mathrm{Zn}$ atom from the $=\mathrm{CH}_{2}$ group, and thereby favors the return of $\mathrm{Cu}^{+}$to $=\mathrm{CH}_{2}$. Overall, the lowest activation energies are observed for the reactions starting from the abundant Int $2 \gamma$ and the (scarcer) Int $2 \alpha$, which lead to products with the (S) configuration in the carbon originally present on the alkyne. Different preferences are seen for the orientation of the acetaldehyde (and consequent configuration on the second stereocenter): the $(S)(S)$ isomer is preferred by $0.5 \mathrm{kcal} \cdot \mathrm{mol}^{-1}$ when the reaction proceeds from Int $2 \alpha$, whereas $(S)(R)$ is preferred by $1.0 \mathrm{kcal} \cdot \mathrm{mol}^{-1}$ when the reaction proceeds from Int $2 \gamma$. This preference correlates with the orientation of the acetaldehyde relative to the substituents of the nonheterosubstituted alkyne bond carbon: the isomer where the hydrogen of acetaldehyde lies above them is consistently preferred due to diminished steric strain.

Production of the products bearing an $(R)$ configuration on the stereocenter arising from the alkyne carbon is much less favorable than those with the opposite configuration (Table 3). The reasons for this behavior are varied: for the reactions starting from Int $2 \beta$, the increase of the transition state energies by $5-7 \mathrm{kcal} \cdot \mathrm{mol}^{-1}$ correlates with the higher proximity of the incoming acetaldehyde with the methyl substituent on the oxazolindinone ring (Figure 11D), whereas for the reactions starting from Int $2 \delta$ the transition state energy is identical to that of the (favored) reactions starting from Int $2 \alpha$, and the large activation energies are instead due to the higher stability of Int $2 \delta$ relative to the energy of the other isoforms. 
A)

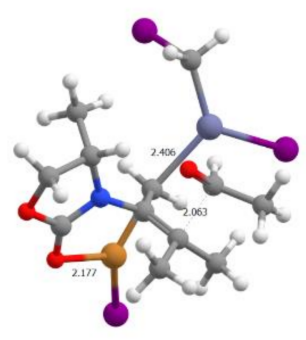

B)

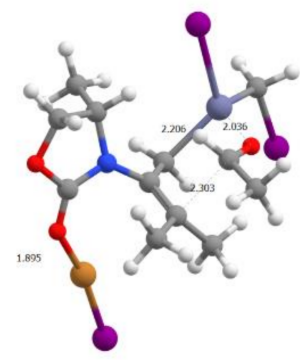

C)

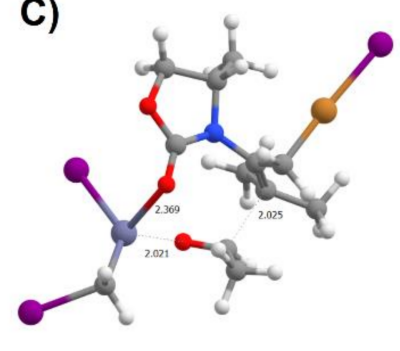

D)

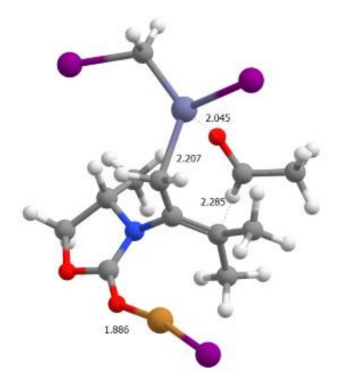

E)

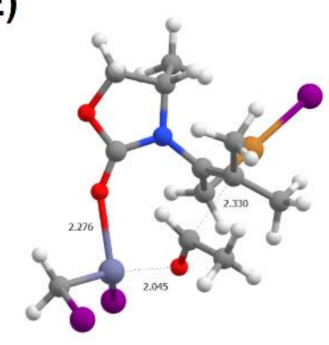

Figure 11. Transition states leading to products with two stereocenters. (A) From Int $2 \alpha$ to the $(S)(S)$ product without $\mathrm{Zn}^{2+}$ complexation by carbonyl; (B) from Int $2 \alpha$ to the $(S)(R)$ product; $(\mathbf{C})$ from Int $2 \gamma$ to the $(S)(R)$ product; (D) from Int2 $\beta$ to the $(R)(S)$ product; (E) from Int2 $\delta$ to the $(R)(S)$ product.

\section{Discussion}

The mechanism arising from our density-functional studies (Figure 12) differs in important respects from the previously postulated reaction sequence. First, the transition state for the postulated carbocupration was shown to lie far above the energies compatible with measurable reaction rates, and the alkylation of the alkyne instead proceeds through direct alkylation of the $\mathrm{Cu}^{+}$-complexed alkyne by the Grignard reagent. Second, the removal of the $\mathrm{MgBr}^{+}$moiety left behind by the Grignard reagent requires the active involvement of the solvent, which entails that eventual modifications of this method must retain the use of a solvent with sufficient $\mathrm{Mg}^{2+}$-chelating ability. Third, the reaction with the zinc carbenoid is not independent of the $\mathrm{Cu}^{+}$catalyst but instead proceeds through methylene transfer to $\mathrm{Cu}^{+}$. This reaction step may, against expectation, occur either with (leading to Int $2 \gamma / \delta$ ) or without (leading to Int $2 \alpha$ ) the assistance of a $\mathrm{Zn}^{2+}$-carbonyl stabilizing interaction. Finally, the stereoselectivity of the carbonyl insertion does not arise from interactions between the aldehyde sidechains and the bulky substituent on the oxazolidinone ring: inspection of the transition states of the most favorable reactions (Figure 11B,C, and Supplementary Materials) shows that the aldehyde actually lies quite far from the oxazolidinone ring. Instead, in both cases (Int $2 \alpha$ and Int $2 \gamma$ ), the disfavored transition state is the one where the bulky substituent on the aldehyde lies immediately above both of the $R_{1}$ and $R_{2}$ substituents present on the other, newly formed, stereocenter. We expect these insights will prove useful for the continuing development of this important methodology and to further increase its scope and stereoselectivity. 


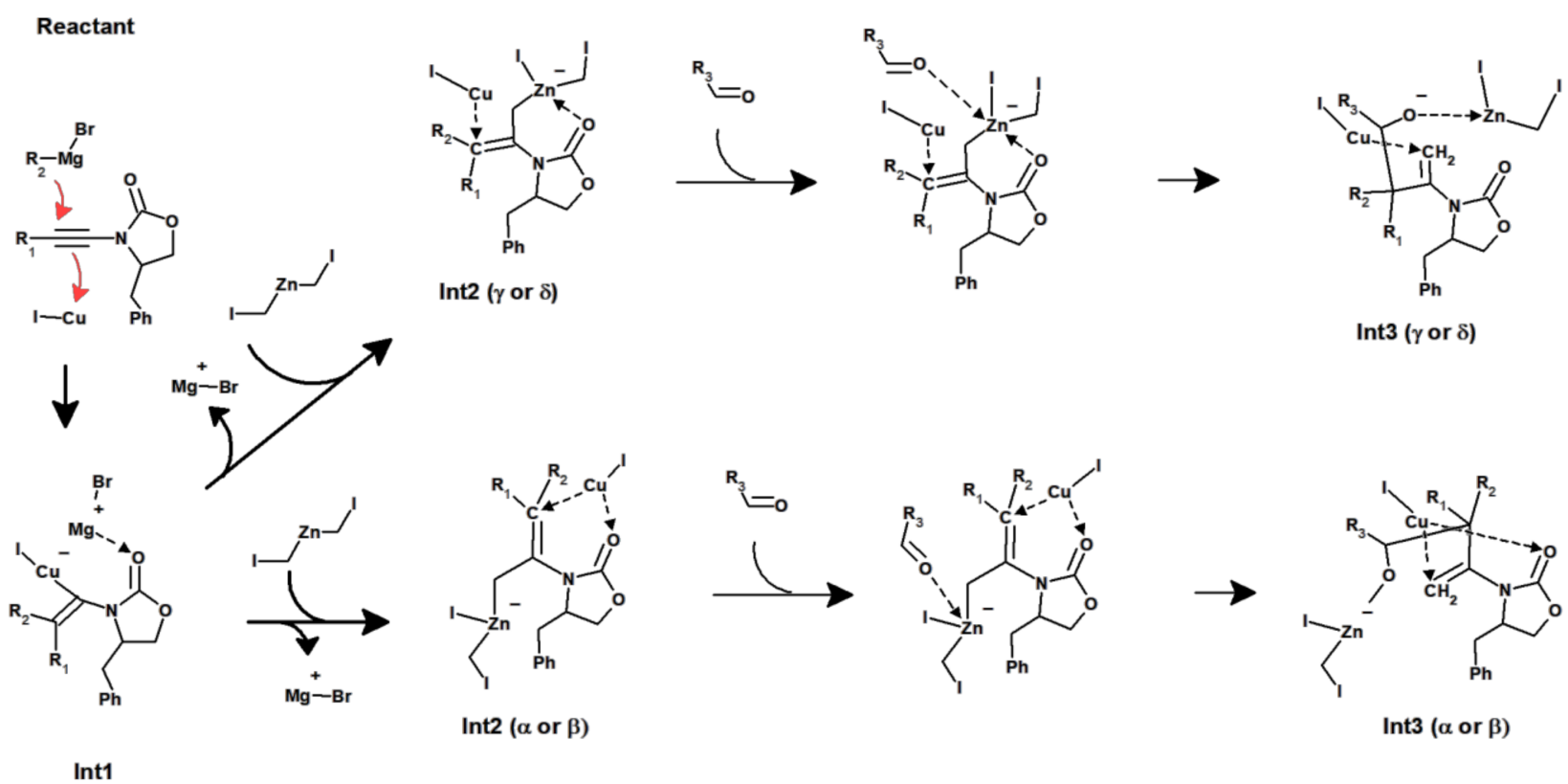

Figure 12. Revised reaction mechanism.

Supplementary Materials: Coordinates of all molecules described in the text, as well as individual energetic data, are available online at https:/ / www.mdpi.com/article/10.3390/app11115002/s1.

Author Contributions: C.E.P.B. and P.J.S. jointly performed the computations. P.J.S. further analyzed the results, conceived and designed the study, and wrote the manuscript. All authors have read and agreed to the published version of the manuscript.

Funding: This work has been financed by FEDER through Programa Operacional Factores de Competitividade-COMPETE and by Portuguese Funds through FCT-Fundação para a Ciência e a Tecnologia under project PTDC/QUI-QUI/111288/2009. This work was also supported by the Applied Molecular Biosciences Unit-UCIBIO, which is financed by national funds from FCT (UIDB/04378/2020).

Data Availability Statement: Full inputs and output files may be downloaded from figshare (DOI: 10.6084/m9.figshare.5481541, accessed date on 27 May 2021).

Conflicts of Interest: The authors declare no conflict of interest.

\section{References}

1. Das, J.P.; Chechik, H.; Marek, I.; Information, S.; Das, J.P.; Chechik, H.; Marek, I.; Stereocenters, Q.; Methods, G. A unique approach to aldol products for the creation of all-carbon quaternary stereocentres. Nat. Chem. 2009, 1, 128-132. [CrossRef] [PubMed]

2. Minko, Y.; Pasco, M.; Lercher, L.; Botoshansky, M.; Marek, I. Forming all-carbon quaternary stereogenic centres in acyclic systems from alkynes. Nature 2012, 490, 522-526. [CrossRef] [PubMed]

3. Perdew, J.P. Unified theory of exchange and correlation beyond the local density approximation. In Electronic Structure of Solids '91; Ziesche, P., Eschrig, H., Eds.; Akademie: Berlin, Germany, 1991; Volume 17, pp. 11-20.

4. Perdew, J.P.; Burke, K.; Ernzerhof, M. Generalized gradient approximation made simple. Phys. Rev. Lett. 1996, 77, 3865-3868. [CrossRef] [PubMed]

5. Bernardo, C.E.P.; Bauman, N.P.; Piecuch, P.; Silva, P.J. Evaluation of density functional methods on the geometric and energetic descriptions of species involved in $\mathrm{Cu}^{+}$-promoted catalysis. J. Mol. Model. 2013, 19, 5457-5467. [CrossRef] [PubMed]

6. Silva, P.J.; Bernardo, C.E.P. Influence of Alkyne and Azide Substituents on the Choice of the Reaction Mechanism of the Cu+-Catalyzed Addition of Azides to Iodoalkynes. J. Phys. Chem. A 2018, 122, 7497-7507. [CrossRef] [PubMed]

7. Baker, J.; Kessi, A.; Delley, B. The generation and use of delocalized internal coordinates in geometry optimization. J. Chem. Phys. 1996, 105, 192-212. [CrossRef]

8. Granovsky, A.A. Firefly 8.0.0. 2013. Available online: http://classic.chem.msu.su/gran/gamess/index.html (accessed on 1 December 2013). 
9. Schmidt, M.W.; Baldridge, K.K.; Boatz, J.A.; Elbert, S.T.; Gordon, M.S.; Jensen, J.H.; Koseki, S.; Matsunaga, N.; Nguyen, K.A.; $\mathrm{Su}, \mathrm{S}$.; et al. General atomic and molecular electronic structure system. J. Comput. Chem. 1993, 14, 1347-1363. [CrossRef]

10. Stevens, W.J.; Krauss, M.; Basch, H.; Jasien, P.G. Relativistic compact effective potentials and efficient, shared-exponent basis sets for the third-, fourth-, and fifth-row atoms. Can. J. Chem. 1992, 70, 612-630. [CrossRef]

11. Swart, M.; Güell, M.; Luis, J.M.; Solà, M. Spin-state-corrected Gaussian-type orbital basis sets. J. Phys. Chem. A 2010, 114, 7191-7197. [CrossRef] [PubMed]

12. Tomasi, J.; Persico, M. Molecular Interactions in Solution: An Overview of Methods Based on Continuous Distributions of the Solvent. Chem. Rev. 1994, 94, 2027-2094. [CrossRef]

13. Mennucci, B.; Tomasi, J. Continuum solvation models: A new approach to the problem of solute's charge distribution and cavity boundaries. J. Chem. Phys. 1997, 106, 5151-5158. [CrossRef]

14. Cossi, M.; Mennucci, B.; Pitarch, J.; Tomasi, J. Correction of cavity-induced errors in polarization charges of continuum solvation models. J. Comput. Chem. 1998, 19, 833-846. [CrossRef]

15. Amovilli, C.; Mennucci, B. Self-consistent-field calculation of Pauli repulsion and dispersion contributions to the solvation free energy in the polarizable continuum model. J. Phys. Chem. B 1997, 5647, 1051-1057. [CrossRef]

16. Sjoberg, P.; Murray, J.S.; Brinck, T.; Politzer, P. Average local ionization energies on the molecular surfaces of aromatic systems as guides to chemical reactivity. Can. J. Chem. 1990, 68, 1440-1443. [CrossRef]

17. Lu, T.; Chen, F. Quantitative analysis of molecular surface based on improved Marching Tetrahedra algorithm. J. Mol. Graph. Model. 2012, 38, 314-323. [CrossRef] [PubMed]

18. Lu, T.; Chen, F. Multiwfn: A multifunctional wavefunction analyzer. J. Comput. Chem. 2012, 33, 580-592. [CrossRef] [PubMed]

19. Mendes, P.; Hoops, S.; Sahle, S.; Gauges, R.; Dada, J.; Kummer, U. Computational modeling of biochemical networks using COPASI. Methods Mol. Biol. 2009, 500, 17-59. [PubMed]

20. Chemla, F.; Ferreira, F. Carbocupration of Alkynes. In PATAI'S Chemistry of Functional Groups; John Wiley \& Sons, Ltd.: Chichester, UK, 2011; pp. 527-584, ISBN 9780470682531.

21. Basheer, A.; Marek, I. Recent advances in carbocupration of $\alpha$-heterosubstituted alkynes. Beilstein J. Org. Chem. 2010, 6, 1-12. [CrossRef] [PubMed] 